\title{
击实粘性土微结构特性的定量评价 ${ }^{*}$ \\ 施 斌王宝军 \\ (南宗人学地球科学系, 南宗 210093) \\ 姜洪涛 \\ (江. 苏教会学院地埋系, 南宗 210013)
}

\section{关键词击实粘性土 微观结构 定量评价}

微观结构是影响击实粘性土工程性质的一个重要因素。在不同含水量下重塑的粘性土在 击实过程中, 其结构不断发生变化, 并直接影响到士的工程性质. 本文应用 Videolab 图悉分 析系统, 对不同含水量下击实粘性土的微观结构进行了定量分析, 提出了 3 种评价上结构定问 性的分量指标, 揭示了粘性土在击实过程中性质变化的内在机理.

\section{1 粘性土微观结构的定量分析}

运用由莫斯科大学研制的 Videolab 图缘分析系统, 可对土微结构的扫描电子显微镜形貌 进行定量分析, 狄得两大部分的定量信息: 第一部分为颗粒或孔隙的形状、大小等特征; 第… 部分为结构单它体的定问特征等。有关获得这些是量信息的基本原理可参见文献 [1 4].

在这些定量分析中, 粘性土微观结构单元体的分向性指标最为重要, 因为它直接影响到粘 性: t的巨程性状. 所谓微观结构单元体在这里主要指的是土的因粒, 而非单一粘土仢物颗 粒. 如们将这些单心体的定量信息转变成反映其定向特征的定量结构参数, 这是值得研究 的. 这里我们提出 3 种有关上结构定向性和有序性的定量指标.

(1)定向性指标一一各向异性率结构分析显示出, 结构单元体在 $0^{\circ} \sim 360^{\circ}$ 上的定问 分布是镜像对称的, 并字椭圆形分布. 为了反映土结构的整体定向性, 我们引进了一个指标 一一各问开性:流, 其定义如下:

$$
I_{u}=\frac{R-r}{R} \times 100 \%,
$$

式中： $I_{a}$ 为各问异性率, $R$ 为椭圆形的长轴长度, $r$ 为椭圆形的短轴长度. $I_{a}$ 取值范国为 $0 \% \sim 100 \%, I_{a}$ 值越大, 上结构的定响性越高.

(2) 们序慛指标一一概率摘我们足义

$$
H_{m}=-\sum_{i=1}^{n} P_{i} \log _{n} P_{i},
$$

$H_{m}$ 为土结构单元体排列的概率嫡. 其中 $P_{i}$ 为结构单元体在某一方位风中现的概率. 在本次研究中, $P_{i}$ 即为在某一方位上单无体的相对足向强度, $n$ 即为在单元体排列方向 $[0 \sim N]$

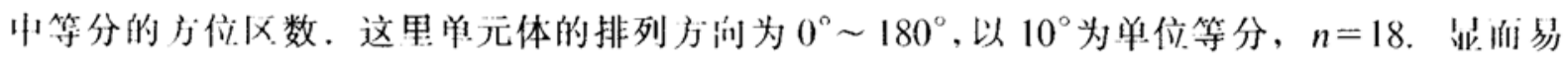

1995-06-05 收椋, 1995-09-25收修收稿

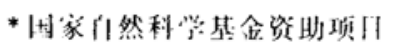


见: $I_{m}$ 的取值范目在 $[0,1], H_{m}$ 越大, 说明结构单无体排列越混乱, 有忬性:降低.

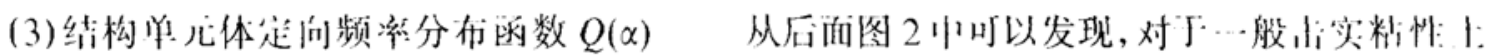

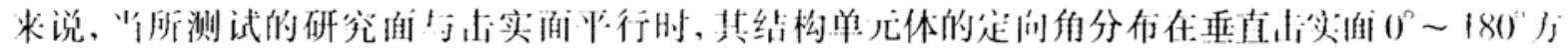

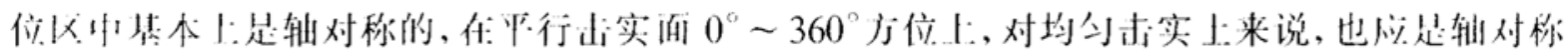

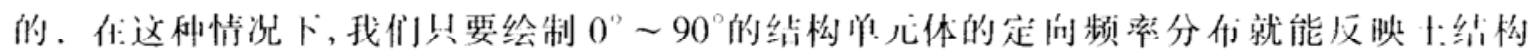

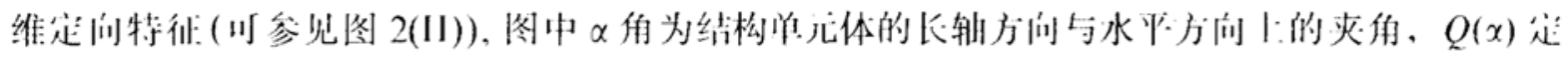

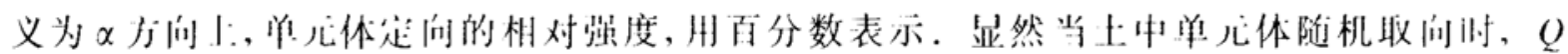
$(\alpha)-\alpha$ 的关系为一条惊行 $\alpha$ 轴的直线.

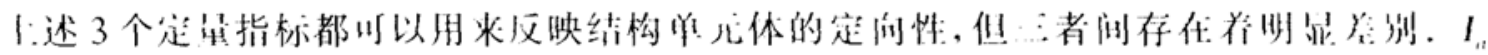

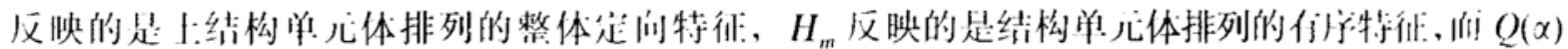
能连续地艮示结构坚儿体的定问特征。

\section{2 击实粘性土中的微结构类型}

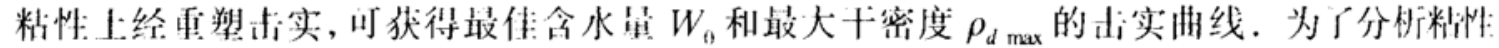
1: 在: li 实过程中的微观结构变化, 我们取湖北勋出胀缩性粘性土为试样, 其物理性质抬标 为: 液限 $\left(W_{l}\right), 55 \%$; 塑性指数 $\left(I_{p}\right), 25 \%$; 小于 $5 \mu \mathrm{m}$ 的粘粒合量, $45 \%$; 主要粘上矿物们管

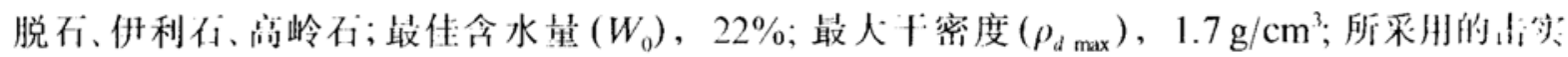

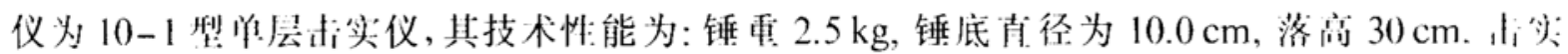
简古行为 $10 \mathrm{~cm}$, 每情功能 $0.225 \mathrm{~kg} / \mathrm{cm}^{3}$. 收其中的一 此，实试样，它们在甫实曲线上的位置见图 1, 用扫 描也 与.监微镜对其微观结构进行观察。总结出了 5 种微 观结构类型, 它们分别代表了山i实曲线 I: 1 5 处试样 的结构特征.这 5 种结构类型是: (1) 松散集粒结构,

（2）紧密集粒结构, (3) 镶误结构, (4) 絮流状结构,

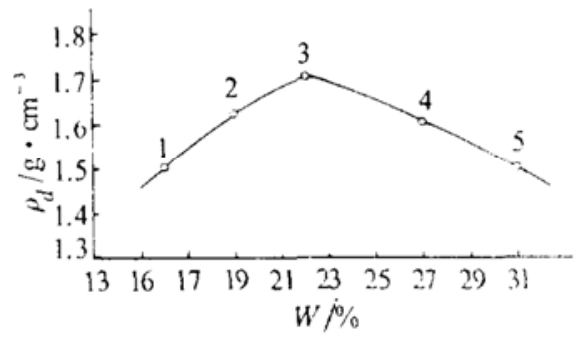

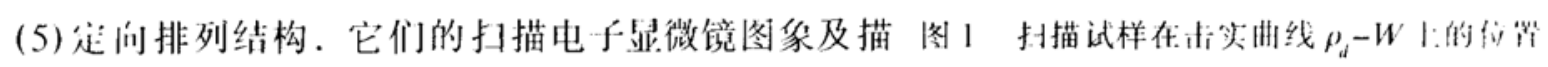
述见文献[5].

\section{5 种微观结构类型的定量评价}

表 1 是 5 种微观结构类型各要素的定量分析结果以及它们的胀缩特继; 图 2 反咉厂这 5 种微观结构学无体分响性的定量分析结果.

表 1 怙实膨胀士微观结构定量分析与胀缩试验结果

\begin{tabular}{|c|c|c|c|c|c|c|c|c|c|c|c|c|}
\hline \multirow{2}{*}{$\begin{array}{l}\vdots 1 \\
\vdots \\
3\end{array}$} & \multirow{2}{*}{$\begin{array}{c}\text { 相对耐积 } \\
\text { (f九防等） }\end{array}$} & \multirow{2}{*}{$\begin{array}{c}\text { 平均孔径 } \\
/ \mu \mathrm{m}\end{array}$} & \multicolumn{4}{|c|}{ 孔行/ $\mu \mathrm{m}$} & \multirow{2}{*}{$\begin{array}{l}\text { 平.均形 } \\
\text { 状系数 }\end{array}$} & \multicolumn{2}{|c|}{ 定们指标 } & \multicolumn{3}{|c|}{ 胀缩性:拮标 } \\
\hline & & & $<0.5$ & $0.5 \sim 5$ & $5 \sim 50$ & $>50$ & & 各们异性冽 & 概呟娧 & $\begin{array}{c}\text { 膨胀力 } \\
/ \mathrm{kPa}\end{array}$ & $\begin{array}{l}\text { 膨胀棌 } \\
50 \mathrm{kPaF}\end{array}$ & 统敢 \\
\hline 1 & 9.2 & 10.5 & 16.7 & 24.7 & 50.2 & 8.4 & 0.57 & 5.1 & 0.98 & 150 & 2.3 & 23 \\
\hline 2 & 7.4 & 5.2 & 21.6 & 38.2 & 40.2 & 0.0 & 0.53 & 4.5 & 0.98 & 505 & 15.3 & 4.2 \\
\hline 3 & 6.5 & 4.2 & 24.4 & 47.5 & 28.1 & 0.0 & 0.49 & 3.6 & 0.97 & 450 & 10.2 & s.o \\
\hline 4 & 6.7 & 4.8 & 23.1 & 51.4 & 25.5 & 0.0 & 0.32 & 2.9 & 0.93 & 18 & 3.5 & 13.1 \\
\hline 5 & 7.2 & 5.7 & 18.8 & 58.4 & 22.8 & 0.0 & 0.20 & 51.9 & 0.85 & 0.0 & -0.5 & 15.2 \\
\hline
\end{tabular}



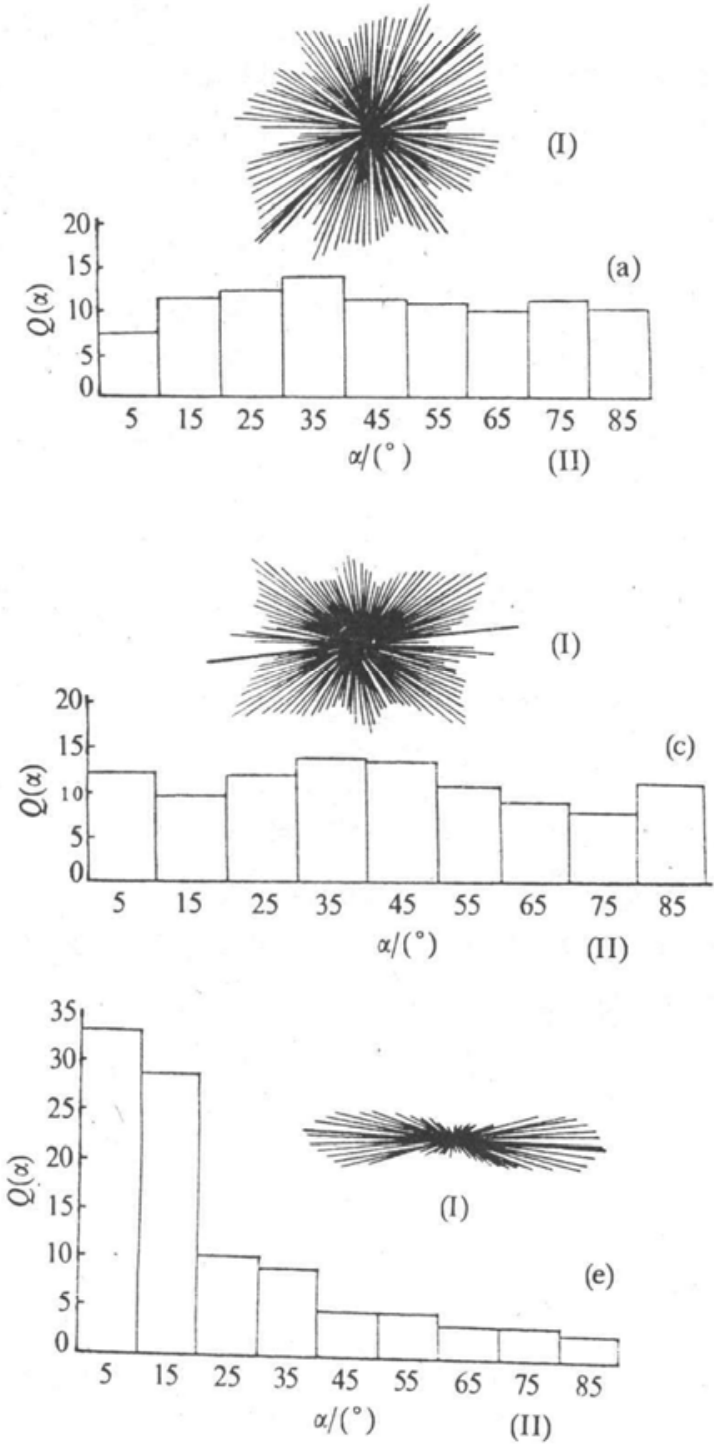
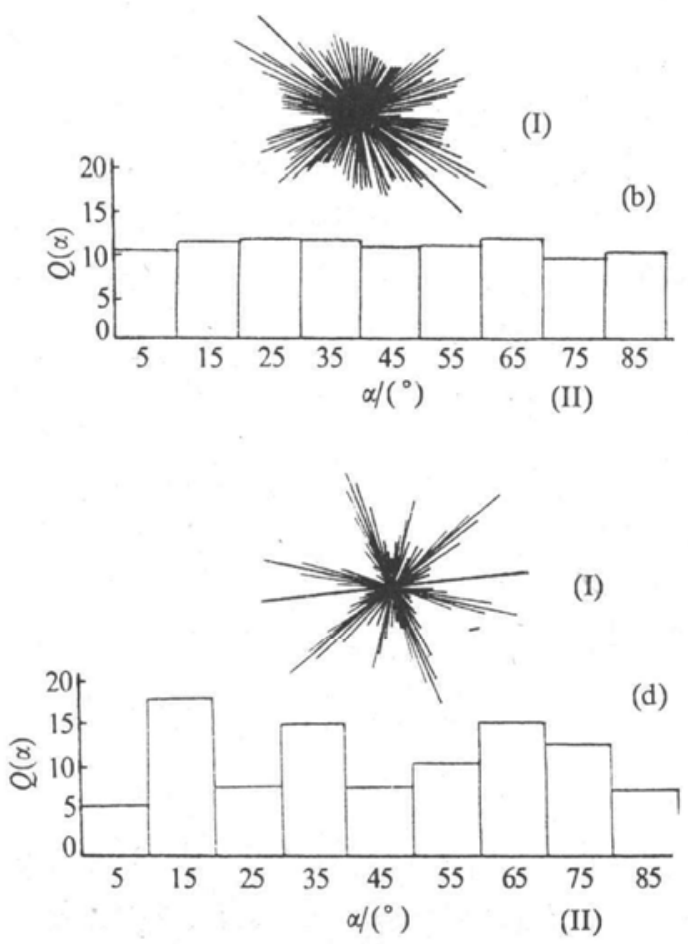

(d)

图 25 种典型微观结构图象定向分析结果 (I) 团粒定向角分布玫瑰图, (II) 团粒定向频率分布直方 图. (a) 松散集粒结构 (在图 1 中位置 1), (b) 紧密集粒 结构 (在图 1 中位置 2), (c) 镶嵌结构 (图 1 中位置 3), (d)紊流状结构 (图 1 中位置4), (e) 定向排列结构(图1 中位置 5)

当 $W \ll W_{0}$ 时, 粘粒扩散层不能充分发展, 粒间的水化膜楔人力较小而吸引力较大, 因 此 团聚体本身结构强度高, 团聚体间作用力强, 土就很难压密. 因而定量结果显示出孔隙大且 混圆, 颗粒无序分布, 各向异性率低, 形成了松散的集聚体结构, 见表 1 和图 2(a). 随着 $W$ 增 大, 孔隙开始变小, 由松散的集粒结构转变成紧密的集粒结构, 土的密度增加, 但微观结构的 定向性和无序性均未得到改善, 见表 1 和图 2(b). 在这一阶段, 虽然含水量不断增加, 但由于 密度也不断增加, 因此土的胀缩性不断增加.

当 $W=W_{0}$ 时, 粘粒扩散层加厚, 粒间水化膜厚度增大, 颗粒间的作用力减小, 团聚体易 于 “滑动”、压密, 一部分含水量较高的团聚体其结构强度减弱, 易于被挤扁, 使粘粒沿某些 颗粒产生定向, 形成镶嵌结构. 表 1 显示孔径变小, 形状开始拉长, 但从 $H_{m}$ 和 $I_{a}$ 指标显示 出, 在最佳含水量处, 土颗粒排列的有序性略有增加, 但基本上还是呈各向同性, 见图 2(c). 在 这一阶段虽然土的密度增加了, 但由于含水量增加, 土的膨胀性开始下降了.

当 $W>W_{0}$ 时, 大量的粘粒扩散层得到高度充分的发展, 其水化膜厚度大大增加, 团聚体 的结构强度大大减弱, 大量的粘粒产生定向, $H_{m}$ 降低, 有序性得到提高, 但 $I_{m}$ 仍较低, 见表 1 
和图 2(d), 形成系流状结构。当 $W \gg W_{0}$ 时, 表 1 显示出在这一阶段孔径开始增加, 孔腙形状 不断拉长, 非状系数只存 $0.2, I_{a}$ 达到 $51.9 \%$, 各问异性明显, $H_{m}$ 降低, 粘粒排列的们序性大 大增大, 见图 2(e), 形成定问排列结构。在这一阶段, 由于含水量不断增加, 上密淁开始卜降, 因洏上的膨胀性降为零, 而收缩性不断增加, 收缩性增加的另一个原因即为上:结构的定问性: 增加了.

上微炶构的上:述变化, 是上具有最佳含水量和最大十密度的标质所住.

\section{4 结论}

(1) Videolab 图像处理系统对获取士微观结构的定量信息指标是十分们用的。

(2) 本文提出的 3 个定量指标都能反映结构单元体的定们性，但三者间存在着明显.

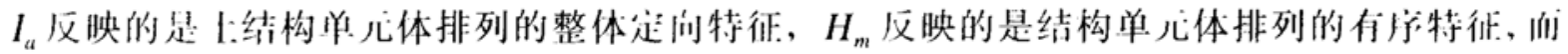
$Q(\alpha)$ 能连续地晨示结构単元体们分们特征.

(3) 重塑粘吽: 上在击实过程中, 随着制备含水量的增加, 其微观结构发生了…系列变化. 作者提识了 5 种结构类型并进行了定量分析.

(4) 在上:的胀缩性与微结构之间存在着良好的关系.

致谢工作中得到了 M.Tolkachev 博士和李生林教授的指导, 作者在此表示衰心感谢.

\section{参考文献}

I Shi Bin, Li Shenglin, Tolkachev M. Quantitative approach on SFM images of microstructure of clay soils. Scieno in ('hina, Series B, 1995, 38(5): 741 748

2 Tovey N K. Krinsley D H. Mapping of the orientation of fine-grained mincral in soils and sediments. Bulletin of IAFG, 1992, 46: $93 \sim 101$

3 Tovey N K. A digital computer technique for orientation analysis of micrographs of soil fabric. J of Microsoopy, 1990), 120: $303 \sim 315$

4 Krinsley D P. Petrographic examination of sedimentary rocks in the SEM using back-scattered electron detectors. J Sed Petr. i984, 54:877 $\sim 888$

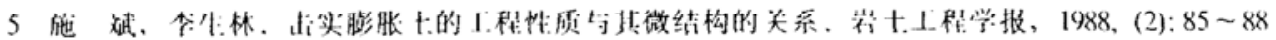

